BARI-TH/95-191

UCT-TP-222/95

January 1995

\title{
Tests of factorization in color suppressed nonleptonic B decay modes
}

\author{
P. Colangelo ${ }^{a}$, C.A. Dominguez ${ }^{b}$, , N. Paver ${ }^{c}$. 苨 \\ ${ }^{a}$ Istituto Nazionale di Fisica Nucleare, Sezione di Bari, Italy \\ ${ }^{b}$ Institute of Theoretical Physics and Astrophysics, University of Cape Town, Rondebosch \\ 7700, South Africa \\ ${ }^{c}$ Dipartimento di Fisica Teorica, Università di Trieste, Italy, and Istituto Nazionale di Fisica \\ Nucleare, Sezione di Trieste, Italy
}

\begin{abstract}
We propose a number of tests of factorization for the color-suppressed nonleptonic decay channels: $B \rightarrow K^{(*)} \eta_{c}$ and $B \rightarrow K^{(*)} \eta_{c}^{\prime}$. The relevant leptonic constants and form factors are calculated within the QCD sum rules approach.
\end{abstract}

\footnotetext{
${ }^{1}$ John Simon Guggenheim Fellow 1994-1995

${ }^{2}$ Also supported by MURST, Ministero dell’Universitá e Ricerca Scientifica e Tecnologica, Italy
} 


\section{Introduction}

It has been recently observed [1, 2] that the available experimental data [3] on the longitudinal polarization of the final particles in the decay $B \rightarrow K^{*} J / \Psi: \rho_{L}=\Gamma(B \rightarrow$ $\left.K^{*} J / \Psi\right)_{L L} / \Gamma\left(B \rightarrow K^{*} J / \Psi\right)=0.84 \pm 0.06 \pm 0.08$, together with the ratio $R_{J / \Psi}=$ $\Gamma\left(B \rightarrow K^{*} J / \Psi\right) / \Gamma(B \rightarrow K J / \Psi)=1.64 \pm 0.34$, can severely constrain the semileptonic $B \rightarrow K^{(*)}$ form factors if these nonleptonic transitions are evaluated within the factorization approach.

Neglecting penguin operators, the relevant effective Hamiltonian density is

$$
\mathcal{H}_{W}=-\frac{G_{F}}{\sqrt{2}} V_{c s}^{*} V_{c b}\left(c_{1}(\mu) O_{1}+c_{2}(\mu) O_{2}\right)+\text { h.c. },
$$

where $O_{1}=\left(\bar{s}_{i} c_{i}\right)_{V-A}\left(\bar{c}_{j} b_{j}\right)_{V-A}$ and $O_{2}=\left(\bar{s}_{i} c_{j}\right)_{V-A}\left(\bar{c}_{j} b_{i}\right)_{V-A}(i, j$ are color indices, $V-A$ refers to $\gamma_{\mu}\left(1-\gamma_{5}\right), G_{F}$ is the Fermi constant and $V_{l m}$ are Cabibbo-Kobayashi-Maskawa matrix elements). The values of the Wilson coefficients at the next-to-leading order in QCD can be taken as $c_{1}\left(m_{b}\right)=1.08 \pm 0.01$ and $c_{2}\left(m_{b}\right)=-0.19 \pm 0.02$ [圈, 5].

The above transitions are color-suppressed. After Fierz reordering Eq. (1), in the framework of factorization and vacuum saturation dominance, the factorized amplitude is

$$
A\left(B \rightarrow K^{(*)} J / \Psi\right)=-\frac{G_{F}}{\sqrt{2}} V_{c s}^{*} V_{c b} a_{2}<K^{(*)}\left|\bar{s} \gamma_{\mu}\left(1-\gamma_{5}\right) b\right| B><J / \Psi\left|\bar{c} \gamma^{\mu}\left(1-\gamma_{5}\right) c\right| 0>
$$

where $a_{2}=c_{2}+c_{1} / N_{c}$, with $N_{c}$ is the number of colors. Eq. (2) involves the matrix element

$$
<J / \Psi(q, \epsilon)\left|\bar{c} \gamma_{\mu} c\right| 0>=f_{J / \Psi} M_{J / \Psi} \epsilon_{\mu}^{*}
$$

where the leptonic constant $f_{J / \Psi}$ is directly obtained from the measured decay $J / \Psi \rightarrow$ $\ell^{+} \ell^{-}$. Equation (2) also involves the semileptonic $B \rightarrow K^{(*)}$ matrix elements $\left(q=p-p^{\prime}\right)$ :巴

$$
<K\left(p^{\prime}\right)\left|\bar{s} \gamma_{\mu} b\right| B(p)>=\left(p+p^{\prime}\right)_{\mu} F_{1}\left(q^{2}\right)+\frac{M_{B}^{2}-M_{K}^{2}}{q^{2}}\left[F_{0}\left(q^{2}\right)-F_{1}\left(q^{2}\right)\right] q_{\mu},
$$

and

\footnotetext{
${ }^{1}$ We adopt the Bauer-Stech-Wirbel parameterization [6] where: $F_{0}(0)=F_{1}(0), A_{3}(0)=A_{0}(0)$ and $A_{3}\left(q^{2}\right)=\left[\left(M_{B}+M_{K^{*}}\right) / 2 M_{K^{*}}\right] A_{1}\left(q^{2}\right)-\left[\left(M_{B}-M_{K^{*}}\right) / 2 M_{K^{*}}\right] A_{2}\left(q^{2}\right)$.
} 


$$
\begin{aligned}
<K^{*}\left(p^{\prime}, \eta\right)\left|\bar{s} \gamma_{\mu}\left(1-\gamma_{5}\right) b\right| B(p)> & =\eta_{\mu \nu \rho \sigma} \eta^{* \nu} p^{\rho} p^{\prime \sigma} \frac{2 V\left(q^{2}\right)}{M_{B}+M_{K^{*}}}-i\left[\left(M_{B}+M_{K^{*}}\right) A_{1}\left(q^{2}\right) \eta_{\mu}^{*}-\right. \\
-\frac{A_{2}\left(q^{2}\right)}{M_{B}+M_{K^{*}}}\left(\eta^{*} \cdot p\right)\left(p+p^{\prime}\right)_{\mu} & \left.-\left(\eta^{*} \cdot p\right) \frac{2 M_{K^{*}}}{q^{2}} q_{\mu}\left(A_{3}\left(q^{2}\right)-A_{0}\left(q^{2}\right)\right)\right] .
\end{aligned}
$$

Actually, in Eq. (2) the form factors must be evaluated at $q^{2}=M_{J / \Psi}^{2}$ and, in particular, the quantities $\rho_{L}$ and $R_{J / \Psi}$ depend on the ratios: $V\left(M_{J / \Psi}^{2}\right) / A_{1}\left(M_{J / \Psi}^{2}\right), A_{2}\left(M_{J / \Psi}^{2}\right) / A_{1}\left(M_{J / \Psi}^{2}\right)$ and $F_{1}\left(M_{J / \Psi}^{2}\right) / A_{1}\left(M_{J / \Psi}^{2}\right)$.

Using predictions of popular theoretical models for the form factors, [2 difficulties are met in simultaneously reproducing the experimental outcomes for $\rho_{L}$ and $R_{J / \Psi}$ [1, 2]. This has prompted a number of interesting phenomenological analyses trying to elucidate the source of the problem. On the one side, the blame could be put on factorization which, in principle, can receive substantial corrections from the nonfactorizable component of Eq. (11), that has the large coefficient $c_{1}$ [9]. At present, such effects are quite difficult to predict by a dynamical calculation.

On the other side, the alternative attitude would be to accept factorization, which is an appealing exact feature of multicolor chromodynamics in the limit $N_{c} \rightarrow \infty$ [10], [] and focus, instead, on form factor predictions. Actually, not all form factor models are quite predictive in the region of large $q^{2} \simeq M_{J / \Psi}^{2}$, and in these cases the $q^{2}$-dependence has to be assumed, e.g., by invoking lowest-lying meson dominance and/or quark counting rules. In this context, assuming factorization, different phenomenological $q^{2}$-dependences have been scrutinized in Ref. [0], attempting to reproduce the available data.

Clearly, experimental studies of other color-suppressed $B$ decays should be very desirable in order to complete the present information and more extensively test the factorization

\footnotetext{
${ }^{2}$ For a rather complete set of references on heavy-to-light meson semileptonic form factors see, e.g., Refs. [1, 8, 8].

${ }^{3}$ Arguments in support of factorization for finite $N_{c}$, in the limit of infinitely heavy quarks, can be found in 11] (with some criticisms in [12), and different analyses of the role of nonfactorizable matrix elements for selected $B$ and $D$ nonleptonic decays [13, 14] do not seem to indicate sizable deviations if $1 / N_{c}$ corrections are collectively discarded 15.
} 
scheme. From this point of view, an interesting role is played by the transitions $B \rightarrow$ $K^{(*)} \eta_{c}$, that differ from the previous ones, $B \rightarrow K^{*} J / \Psi$, just by the charm-quark spin configuration. These processes, not measured yet, have been considered also in the phenomenological analyses of Refs. [7, 8], where relations between the branching ratios of $B \rightarrow K^{(*)} \eta_{c}$ and $B \rightarrow K^{(*)} J / \Psi$ in terms of ratios of leptonic constants and form factors have been worked out.

In this note, we consider predictions for $B \rightarrow K^{(*)} \eta_{c}$ and $B \rightarrow K^{(*)} \eta_{c}^{\prime}$ that can be obtained in the factorization approximation using the method of QCD sum rules. The aim of our discussion differs from [7, 8 in that we adopt here a specific theoretical framework to estimate consistently from the outset all nonperturbative quantities necessary to predict these processes. Indeed, in this case the relevant leptonic constants in Eq. (22), where $J / \Psi$ must be replaced by $\eta_{c}$ or $\eta_{c}^{\prime}$, are

$$
<\eta_{c}\left(\eta_{c}^{\prime}\right)\left|\bar{c} \gamma_{\mu} \gamma_{5} c\right| 0>=-i f_{\eta_{c}\left(\eta_{c}^{\prime}\right)} q_{\mu},
$$

that are not experimentally known, but must be determined theoretically. Previous references rely on constituent quark model arguments to relate $f_{\eta_{c}}$ to $f_{J / \Psi}$. In fact, leptonic constants represent a natural field of application for QCD sum rules [16]. The virtue of this approach, being fully relativistic and field-theoretic by construction, is that it allows to avoid the notion of confined-quark wavefunction, and it incorporates naturally fundamental features of $\mathrm{QCD}$, such as perturbative asymptotic freedom and nonperturbative quark and gluon condensation. Thus, as a computational scheme alternative to the constituent quark model, it should be sensible to present an updated estimate of $f_{\eta_{c}}$ and $f_{\eta_{c}^{\prime}}$ in the framework of QCD sum rules. We recall that besides their relevance to the $B$ decays under consideration, emphasized in Ref. [17], these constants are of phenomenological interest also to other applications of QCD sum rules, such as the radiative processes $J / \Psi \rightarrow \eta_{c} \gamma$ and $\eta_{c}\left(\eta_{c}^{\prime}\right) \rightarrow \gamma \gamma[18 \rrbracket$.

The other significant aspect of the present calculation is that the needed $B \rightarrow K^{(*)}$ form factors $F_{0}\left(q^{2}\right)$ and $A_{0}\left(q^{2}\right)$ (see Eqs. (4) and (55)) have not been studied as extensively as 
others. Since these form factors must be evaluated at $q^{2}=M_{\eta_{c}}^{2}$ or $q^{2}=M_{\eta_{c}^{\prime}}^{2}$, we can exploit the capability of QCD sum rules to provide an evaluation of their $q^{2}$-dependence, within some theoretical uncertainty. This would complete the phenomenological description of color-suppressed $B$ decays to charmonium based on QCD sum rules and factorization, by the addition of a set of predictions which, ultimately, could be interesting to compare to experimental data (when available) or to predictions from alternative nonperturbative schemes.

The paper is organized as follows. In Sec. 2 we present a QCD sum rule calculation of the leptonic constants $f_{\eta_{c}}$ and $f_{\eta_{c}^{\prime}}$, together with a comparison with previous determinations. In Sec. 3 we briefly describe a calculation of the form factors $F_{0}\left(q^{2}\right)$ and $A_{0}\left(q^{2}\right)$ for the transitions $B \rightarrow K, K^{*}$; finally, in Sec. 4 we give predictions for ratios such as $\Gamma\left(B \rightarrow K^{*} \eta_{c}^{\prime}\right) / \Gamma\left(B \rightarrow K^{*} \eta_{c}\right)$ and $\Gamma\left(B \rightarrow K \eta_{c}^{\prime}\right) / \Gamma\left(B \rightarrow K \eta_{c}\right)$.

\section{QCD sum rule calculation of $f_{\eta_{c}}$ and $f_{\eta_{c}^{\prime}}$}

In order to determine the leptonic constants of the $\eta_{c}$ and $\eta_{c}^{\prime}$ mesons from QCD sum rules we consider the two-point function

$$
\psi_{5}(q)=i \int d^{4} x e^{i q x}<0\left|T\left(\partial^{\mu} A_{\mu}(x) \partial^{\nu} A_{\nu}^{\dagger}(0)\right)\right| 0>
$$

where $\partial^{\mu} A_{\mu}(x)=2 m_{c}: \bar{c}(x) i \gamma_{5} c(x):$. This two-point function is known in perturbative QCD to two-loop order [19], and the leading non-perturbative term in the Operator Product Expansion (OPE) is also known [16] (it is given in terms of the gluon condensate). We exploit two different types of QCD sum rules, viz. Hilbert transforms at $Q^{2}=0$, and Laplace transforms. The former can be written as

$$
M_{n}(0) \equiv \frac{1}{\pi} \int_{0}^{\infty} \frac{d s}{s^{n+2}} \operatorname{Im} \psi_{5}(s) .
$$

The perturbative QCD contribution is given by [19]

$$
\left.M_{n}(0)\right|_{P T}=\frac{3}{4 \pi^{2}} \frac{\Gamma^{2}(n)}{\Gamma(2 n)} \frac{1}{(2 n+1)}\left(\frac{1}{m_{c}^{2}}\right)^{n-1}\left(1+a_{n} \alpha_{s}\right),
$$


where

$$
\begin{gathered}
a_{n}=\frac{4}{3 \pi}\left\{1-\frac{1}{2 n}-\frac{3}{n+1}-\frac{3}{2(n+2)}+\left(\frac{1}{n}+\frac{1}{n+1}\right)\right. \\
\left.\times \sum_{r=1}^{n+1}\left[\frac{n+2}{r} \frac{B\left(r, \frac{1}{2}\right)}{B\left(n, \frac{1}{2}\right)}-\frac{3}{r}+\frac{2}{2 r-1}\right]\right\},
\end{gathered}
$$

and $B(x, y)$ is the beta function. The leading non-perturbative term in the OPE gives the following contribution 16

$$
\left.M_{n}(0)\right|_{N P}=-\frac{1}{24}<\frac{\alpha_{s}}{\pi} G^{2}>\frac{1}{\left(4 m_{c}^{2}\right)^{n+1}} \frac{(n-3) \Gamma\left(\frac{1}{2}\right) \Gamma(n+3)}{\Gamma(n+5 / 2)} .
$$

The hadronic spectral function is parametrized by the standard ansatz

$$
\left.\frac{1}{\pi} \operatorname{Im} \psi_{5}(s)\right|_{H A D}=f_{\eta_{c}}^{2} M_{\eta_{c}}^{4} \delta\left(s-M_{\eta_{c}}^{2}\right)+\left.\theta\left(s-s_{0}\right) \frac{1}{\pi} \operatorname{Im} \psi_{5}(s)\right|_{P T},
$$

where a second pole term for the $\eta_{c}^{\prime}$ may be added to Eq. (12). Putting everything together, and ignoring as a first step the contribution of the $\eta_{c}^{\prime}$ meson, the Hilbert transform QCD sum rules become

$$
\begin{aligned}
\frac{f_{\eta_{c}}^{2}}{M_{\eta_{c}}^{2 n}}=\int_{4 m_{c}^{2}}^{s_{0}} \frac{d s}{s^{n+2}} & \left.\frac{1}{\pi} \operatorname{Im} \psi_{5}(s)\right|_{P T}-\frac{1}{24}<\frac{\alpha_{s}}{\pi} G^{2}>\frac{1}{\left(4 m_{c}^{2}\right)^{n+4}} \\
& \times \frac{(n-3) \Gamma\left(\frac{1}{2}\right) \Gamma(n+3)}{\Gamma(n+5 / 2)} .
\end{aligned}
$$

We take ratios of two consecutive moments for various values of $n$ in order to find the duality window (range of values of $s_{0}$ ) for which the mass of the $\eta_{c}$ is correctly predicted. Once this mass is accounted for, the leptonic decay constant follows from any given moment. In addition to expecting some region of stability in $s_{0}$, one also expects the mass and decay constant to be reasonably independent of $n$. These expectations are partly fulfilled by the results of our calculation, as may be appreciated from Figs. 1 and 
2 where we show $M_{\eta_{c}}$ and $\tilde{f}_{\eta_{c}}=f_{\eta_{c}} / \sqrt{2}$ as a function of $n$ for the particular choice: $\Lambda=200 \mathrm{MeV}, m_{c}=1.39 \mathrm{GeV}$, and $<\alpha_{s} G^{2}>=0.063 \mathrm{GeV}^{4}$. The solid (dash) curve in Fig. 1 is the theoretical (experimental) result. In Fig. 2 the solid (dash) curve is obtained by using the theoretical (experimental) value of the $\eta_{c}$ mass in the Hilbert moments. While the stability in $n$ is more than adequate, there is some sensitivity to changes in $s_{0}$. This feature is well known from applications of QCD sum rules to heavy-light quark systems. The situation here, in the case of two heavy quarks, is then not much different. We have explored the following range of parameters, dictated by the gluon condensate and quark-mass analyses of [20] and [21], respectively: $\left\langle\alpha_{s} G^{2}>=0.063-0.19 \mathrm{GeV}^{4}\right.$, $m_{c}=1.46 \pm 0.07 \mathrm{GeV}$. The QCD scale was varied in the range $\Lambda=200-300 \mathrm{MeV}$. The values of $s_{0}$ needed to correctly reproduce the experimental value of $M_{\eta_{c}}$ were found in the range $s_{0}=9.5-10.5 \mathrm{GeV}^{2}$. Results for $M_{\eta_{c}}$ and $f_{\eta_{c}}$ corresponding to various choices of $\Lambda, m_{c}$, the gluon condensate and $s_{0}$ are qualitatively similar to Figs. 1 and 2 . Quantitatively, we find $f_{\eta_{c}} \simeq 256-300 \mathrm{MeV}$.

In a second step, we include a second pole in the spectral function Eq. (12) to account for the $\eta_{c}^{\prime}$ meson, and repeat the analysis. We find that, in order to reproduce correctly the experimental values of both $M_{\eta_{c}}$ and $M_{\eta_{c}^{\prime}}$ (for the mass of $\eta_{c}^{\prime}$ we use the value $M_{\eta_{c}^{\prime}}=$ $3595 \pm 5 \mathrm{MeV}$ [22]), the value of $s_{0}$ must increase by some $30 \%\left(s_{0} \simeq 13-14 \mathrm{GeV}^{2}\right)$. This increase is to be expected, since now $s_{0} \geq M_{\eta_{c}^{\prime}}^{2}$. We find:

$$
f_{\eta_{c}} \simeq 301-326 \mathrm{MeV}, \quad f_{\eta_{c}^{\prime}} \simeq 231-255 \mathrm{MeV}
$$

and, therefore, the result for $f_{\eta_{c}}$ is now somewhat higher. The reason why the uncertainty in $f_{\eta_{c}}$ is now smaller than before is easy to understand. With a two-pole spectral function, the sum rules are now constrained to reproduce correctly the two meson masses simultaneously. This constraint effectively reduces the size of the parameter space spanned by $\Lambda, m_{c},<\alpha_{s} G^{2}>$ and $s_{0}$. 
Next, we make use of the Laplace transform QCD sum rule and its first derivative 23]

$$
\begin{gathered}
f_{\eta_{c}}^{2} M_{\eta_{c}}^{4} e^{-M_{\eta_{c}}^{2} / M_{L}^{2}}=\left.\int_{4 m_{c}^{2}}^{s_{0}} d s e^{-s / M_{L}^{2}} \frac{1}{\pi} \operatorname{Im} \psi_{5}(s)\right|_{P T} \\
-\frac{m_{c}^{2}}{6 \sqrt{\pi}}<\alpha_{s} G^{2}>e^{-4 m_{c}^{2} / M_{L}^{2}}\left[G\left(-\frac{3}{2}, \frac{3}{2}, \frac{4 m_{c}^{2}}{M_{L}^{2}}\right)-6 G\left(-\frac{1}{2}, \frac{3}{2}, \frac{4 m_{c}^{2}}{M_{L}^{2}}\right)\right] \\
f_{\eta_{c}}^{2} M_{\eta_{c}}^{6} e^{-M_{\eta_{c}}^{2} / M_{L}^{2}}=\left.\int_{4 m_{c}^{2}}^{s_{0}} d s s e^{-s / M_{L}^{2}} \frac{1}{\pi} \operatorname{Im} \psi_{5}(s)\right|_{P T} \\
-4 m_{c}^{2} \frac{m_{c}^{2}}{6 \sqrt{\pi}}<\alpha_{s} G^{2}>e^{-4 m_{c}^{2} / M_{L}^{2}}\left\{G\left(-\frac{3}{2}, \frac{3}{2}, \frac{4 m_{c}^{2}}{M_{L}^{2}}\right)\right. \\
\left.-6 G\left(-\frac{1}{2}, \frac{3}{2}, \frac{4 m_{c}^{2}}{M_{L}^{2}}\right)-\frac{3}{2} G\left(-\frac{1}{2}, \frac{3}{2}, \frac{4 m_{c}^{2}}{M_{L}^{2}}\right)+3 G\left(\frac{1}{2}, \frac{3}{2}, \frac{4 m_{c}^{2}}{M_{L}^{2}}\right)\right\}
\end{gathered}
$$

where $M_{L}^{2}$ is the Laplace variable and the function $G(b, c, \omega)$

$$
G(b, c, \omega)=\frac{\omega^{-b}}{\Gamma(c)} \int_{0}^{\infty} d t t^{c-1} e^{-t}\left(1+\frac{t}{\omega}\right)^{-b}
$$

is related to the Whittaker function $W_{\lambda, \mu}(\omega)$ through 24]

$$
G(b, c, \omega)=\omega^{\mu-\frac{1}{2}} e^{\omega / 2} W_{\lambda}, \mu(\omega)
$$

Considering first a one-pole spectral function, the ratio of Eqs. (16) and (15) determines $M_{\eta_{c}}$ as a function of the Laplace variable $M_{L}^{2}$ and of $s_{0}$. We find that $M_{\eta_{c}}$ is stable against changes in $M_{L}^{2}$ in the wide region $M_{L}^{2} \simeq 1-5 \mathrm{GeV}^{2}$. The experimental value of $M_{\eta_{c}}$ is correctly reproduced for $s_{0} \simeq 10 \mathrm{GeV}^{2}$, roughly independent of the values of $\Lambda, m_{c}$ and $<\alpha_{s} G^{2}>$. This value of $s_{0}$ is in agreement with the one obtained from the Hilbert moments. In Fig. 3 we show the result for $M_{\eta_{c}}$ (solid line) compared to the experimental value (dash line), for $\Lambda=200 \mathrm{MeV}, m_{c}=1.39 \mathrm{GeV}$ and $<\alpha_{s} G^{2}>=0.063 \mathrm{GeV}^{4}$. The 
result for $\tilde{f}_{\eta_{c}}=f_{\eta_{c}} / \sqrt{2}$ obtained by using the predicted (experimental) value of $M_{\eta_{c}}$ is shown in Fig. 4 as the solid (dash) curve. Exploring the parameter space of $\Lambda, m_{c}$, $<\alpha_{s} G^{2}>$ and $s_{0}$ leads to the prediction: $f_{\eta_{c}} \simeq 265-274 \mathrm{MeV}$, in agreement with the Hilbert moments.

Adding a second pole to the hadronic spectral function, considering higher derivatives of Eq. (15) in $1 / M_{L}^{2}$, and redoing the analysis we find that it is possible to reproduce the experimental values of both $M_{\eta_{c}}$ and $M_{\eta_{c}^{\prime}}$ for $s_{0} \simeq 13-14 \mathrm{GeV}^{2}$. At the same time, the stability region in the Laplace variable $M_{L}^{2}$ remains wide: $M_{L}^{2} \simeq 2-5 \mathrm{GeV}^{2}$. The predictions for the leptonic decay constants are

$$
f_{\eta_{c}} \simeq 292-310 \mathrm{MeV}, \quad f_{\eta_{c}^{\prime}} \simeq 247-269 \mathrm{MeV},
$$

in agreement with the results from the Hilbert moments, Eq. (14). Combining the predictions from the two methods we obtain our final result

$$
\begin{gathered}
f_{\eta_{c}}=309 \pm 17 \mathrm{MeV}, \quad f_{\eta_{c}^{\prime}}=250 \pm 19 \mathrm{MeV}, \\
\frac{f_{\eta_{c}^{\prime}}}{f_{\eta_{c}}}=0.8 \pm 0.1 .
\end{gathered}
$$

Estimates of $f_{\eta_{c}}, f_{\eta_{c}^{\prime}}$, and of the $J / \Psi$ leptonic constant were made many years ago, in the early days of the QCD sum rules approach [25, 26, 27]. For example, in [25] a QCD sum rule determination of $f_{\eta_{c}}$ has been carried out at a one-loop approximation in perturbative QCD, and no gluon condensate corrections, in the framework of Hilbert moments. With an input value of the charm quark mass: $m_{c}=1.25 \mathrm{GeV}$ (to be compared with the recent determination 21] employed here: $m_{c}=1.46 \pm 0.07 \mathrm{GeV}$ ), and the choice $s_{0}=16 \mathrm{GeV}^{2}$, the value $f_{\eta_{c}}=252-369 \mathrm{MeV}$ has been obtained. Our determination here represents an improvement in many respects: radiative (two-loop) as well as non-perturbative corrections have been incorporated into the theoretical side of the sum rules, Hilbert and Laplace sum rules have been employed, updated values of the input parameters have been adopted, and the analysis was constrained to reproduce correctly the masses of both pseudoscalar mesons: $M_{\eta_{c}}$ and $M_{\eta_{c}^{\prime}}$. 


\section{3. $F_{0}\left(q^{2}\right)$ and $A_{0}\left(q^{2}\right)$ from QCD sum rules}

The form factors $F_{0}\left(q^{2}\right)$ and $A_{0}\left(q^{2}\right)$, related to the weak transitions $B \rightarrow K$ and $B \rightarrow K^{*}$, can be computed by three-point function QCD sum rules, following the same strategy adopted in the calculation of the leptonic constants $f_{\eta_{c}}$ and $f_{\eta_{c}^{\prime}}$. The starting point is provided by the correlators [28, 29]:

$$
\Pi_{\mu \nu}\left(p, p^{\prime}, q\right)=i^{2} \int d x d y e^{i\left(p^{\prime} \cdot x-p \cdot y\right)}<0\left|T\left\{j_{\nu}^{K}(x) V_{\mu}(0) j_{5}^{\dagger}(y)\right\}\right| 0>
$$

and

$$
T_{\mu \nu}\left(p, p^{\prime}, q\right)=i^{2} \int d x d y e^{i\left(p^{\prime} \cdot x-p \cdot y\right)}<0\left|T\left\{j_{\nu}^{K^{*}}(x) A_{\mu}(0) j_{5}^{\dagger}(y)\right\}\right| 0>;
$$

the quark currents $j_{5}$ and $j_{\nu}^{K, K^{*}}$ are given by: $j_{5}=\bar{d} i \gamma_{5} b, j_{\nu}^{K}=\bar{d} \gamma_{\nu} \gamma_{5} s, j_{\nu}^{K^{*}}=\bar{d} \gamma_{\nu} s$, whereas the flavour changing weak currents $V$ and $A$ are: $V_{\mu}=\bar{s} \gamma_{\mu} b$ and $A_{\mu}=\bar{s} \gamma_{\mu} \gamma_{5} b$.

The products: $q^{\mu} \Pi_{\mu \nu}$ and $q^{\mu} T_{\mu \nu}$ receive a hadronic contribution from the states $B, K$ and $B, K^{*}$, respectively, so that they can be expressed in terms of the form factors $F_{0}\left(q^{2}\right)$ and $A_{0}\left(q^{2}\right)$ and of a continuum of states; on the other hand, a QCD calculation can be performed for the same operator products, in the limit of large and spacelike $p^{2}$ and $p^{\prime 2}$, in terms of the perturbative QCD contribution and non-perturbative power corrections, proportional to vacuum matrix elements of high dimensional operators. The matching of the hadronic and QCD representations of the correlators can be improved by a double Borel transform in the variables $-p^{2}$ and $-p^{\prime 2}$. We omit here the details of the calculation,

and simply present the result of such a procedure for the form factor $F_{0}\left(q^{2}\right)$. Including power corrections in the OPE up to dimension 5 condensates, one obtains the following equation:

$$
\begin{aligned}
f_{K} f_{B} \frac{M_{B}^{2}}{m_{b}}\left(M_{B}^{2}-M_{K}^{2}\right) F_{0}\left(q^{2}\right) & =\frac{3}{8 \pi^{2}} \int_{D} d s d s^{\prime} \rho\left(s, s^{\prime}, q^{2}\right) \exp \left[\frac{M_{B}^{2}-s}{M^{2}}+\frac{M_{K}^{2}-s^{\prime}}{M^{\prime 2}}\right] \\
-\left(m_{b}-m_{s}\right)\left\{m_{b}<\bar{q} q>\right. & \left.-d_{5}<\bar{q} g_{s} \sigma^{\mu \nu} G_{\mu \nu}^{a} \frac{\lambda^{a}}{2} q>\right\} \exp \left[\frac{M_{B}^{2}-m_{b}^{2}}{M^{2}}+\frac{M_{K}^{2}-m_{s}^{2}}{M^{\prime 2}}\right]
\end{aligned}
$$


where the spectral function $\rho$ is given by:

$$
\begin{gathered}
\rho\left(s, s^{\prime}, q^{2}\right)=\frac{1}{\sqrt{\lambda}}\left\{\left(m_{b}-m_{s}\right) \Delta+\frac{\left(\Delta u-2 \Delta^{\prime} s\right)}{\lambda}\left[m_{b}\left(-2 \Delta+2 \Delta^{\prime}+u-2 s^{\prime}\right)+m_{s}(2 s-u)\right]\right\} \\
\left(\Delta=s-m_{b}^{2}, \Delta^{\prime}=s^{\prime}-m_{s}^{2}, u=s+s^{\prime}-q^{2} \text { and } \lambda=u^{2}-4 s s^{\prime}\right) . \text { The coefficient } d_{5} \text { reads: } \\
d_{5}=\frac{m_{b}^{2} m_{s}}{4 M^{\prime}}-\frac{\left(m_{b}-m_{s}\right)}{6 M^{\prime 2}}+\frac{2 m_{b}}{3 M^{2}}+\frac{m_{b}^{3}}{4 M^{4}}+\frac{m_{b}\left(m_{b}^{2}+m_{s}^{2}-m_{b} m_{s}-q^{2}\right)}{6 M^{2} M^{\prime 2}} .
\end{gathered}
$$

The integration region $D$ in Eq. (24) is limited by the effective thresholds $s_{0}$ and $s_{0}^{\prime}$ separating, in the sum rule, the contribution of the ground state from the hadronic continuum, the latter being modeled by the leading order perturbative QCD. The parameters employed in the calculation are the quark masses: $m_{b}=4.6 \mathrm{GeV}$ [30] and $m_{s}=$ $0.175 \pm 0.020 \mathrm{GeV}$ 31 (we neglect up and down quark masses), the leptonic constants $f_{B}=0.18 \mathrm{GeV}$ [32] and $f_{K}=0.16 \mathrm{GeV}$. As for the dimension 3 and dimension 5 condensates, we take $<\bar{q} q>(1 \mathrm{GeV})=(-230 \mathrm{MeV})^{3}$ and $<\bar{q} g_{s} \sigma^{\mu \nu} G_{\mu \nu}^{a} \frac{\lambda^{a}}{2} q>=m_{0}^{2}<\bar{q} q>$, with $m_{0}^{2}=0.8 \mathrm{GeV}^{2}$; within the final uncertainties, rescaling the quark and mixed condensate to higher scales by the leading-log approximation of the anomalous dimensions does not affect the numerical results for the form factors.

To derive $F_{0}$ from Eq. (24), one looks for a region where the result is stable under variation of the Borel parameters $M^{2}, M^{\prime 2}$, and of the continuum thresholds $s_{0}$ for the $B$-channel and $s_{0}^{\prime}$ for the $K$-channel. We find that stability is obtained by choosing the continuum thresholds in the ranges $s_{0}=33-36 \mathrm{GeV}^{2}, s_{0}^{\prime}=1.3-1.5 \mathrm{GeV}^{2}$, and the Borel parameters in the range $M^{2}=8 \pm 1 \mathrm{GeV}^{2}$ and $M^{\prime 2}=2.0 \pm 0.4 \mathrm{GeV}^{2}$. Let us finally notice that the OPE expansion of the correlators in Eqs. (22) and (23), starting from spacelike values of the momentum transferred, can be extrapolated to positive $q^{2}$ provided that one is far from non-Landau singularities [33. We compute the form factors up to $q^{2}=15 \mathrm{GeV}^{2}$, which is in the safe region.

The result for $F_{0}\left(q^{2}\right)$ corresponding to the above input numbers is depicted in Fig. 5 .

The analogous expressions relevant to $A_{0}\left(q^{2}\right)$ can be found in Ref. [34]. In this case, the 
same input parameters are used as for $F_{0}\left(q^{2}\right)$, except that we need, in this case, $f_{K^{*}}=$ $0.22 \mathrm{GeV}$ and the threshold in the $K^{*}$-channel is now in the range $s_{0}^{\prime}=1.5-1.7 \mathrm{GeV}^{2}$ (while the range of $s_{0}$ for the $B$-channel is the same as before). The results for $A_{0}\left(q^{2}\right)$ are displayed in Fig. 6.

As an important virtue of this calculation, we remark that for both $A_{0}$ and $F_{0}$ there exists, in the sum rules, a hierarchical structure in the OPE expansion, namely the leading perturbative term is numerically larger than the power corrections. This is a particularly welcomed feature of the present calculation, from the point of view of this theoretical framework, in comparison e.g. with the analogous determination of the form factor $A_{2}\left(q^{2}\right)$.

Turning to a discussion of the results, we observe in $A_{0}\left(q^{2}\right)$ a sharp increase when $q^{2}$ varies in the range $q^{2}=0-15 \mathrm{GeV}^{2}$. The form factor can be fitted by the expression:

$$
A\left(q^{2}\right)=\frac{A(0)}{1-\frac{q^{2}}{M_{P}^{2}}}
$$

with $A_{0}(0)=0.27 \pm 0.03$ and $M_{P}=4.8 \pm 0.2 \mathrm{GeV}$. Therefore, the mass of the pole in $A_{0}$ is slightly smaller than the mass of the first singularity in the $q^{2}$ channel: $M_{B_{s}}=$ $5.375 \pm 0.006 \mathrm{GeV}$. This behaviour is similar to what has been observed in Ref. [34] for the channels $D \rightarrow K^{*}, D \rightarrow \rho$, and $B \rightarrow \rho$.

On the other hand, the $q^{2}$-dependence of $F_{0}\left(q^{2}\right)$ is rather soft. We obtain $F_{0}(0)=0.29 \pm$ 0.03, whereas the fitted mass of the pole in Eq. (27) is $M_{P} \simeq 7.5 \mathrm{GeV}$, to be compared to the mass of the first resonance in the $q^{2}$ channel, a $b \bar{s}\left(0^{+}\right)$state expected, in constituent quark models, in the region near $6 \mathrm{GeV}$ (in the BWS model the value: $M_{(b \bar{s})}\left(0^{+}\right)=$ $5.89 \mathrm{GeV}$ is chosen for the mass of this state). This behaviour is analogous to that observed for the form factor $F_{0}\left(q^{2}\right)$ in $B \rightarrow \pi$, computed in the framework of the infinite heavy quark mass limit in Ref. [35].

These results support the observation, made in Ref. [7], that the assumption of a given $q^{2}$-dependence (such as polar, multipolar, etc.) in heavy meson semileptonic form factors 
should be more safely confirmed by some explicit theoretical calculation. In fact, although the dominance of a singularity is reasonable for high values of $q^{2}$, it is possible that, for lower $q^{2}$, the contribution of higher states or of different dynamical mechanisms modifies the polar behaviour. The same argument holds for the application of QCD counting rules, that a priori are rigorous in the limit of large, spacelike $q^{2}$.

\section{Predictions and conclusions}

In the constituent quark model the leptonic constants of the charmonium system can be expressed in terms of the $c \bar{c}$ wave function at the origin $\Psi(0)$ [17:

$$
\begin{gathered}
f_{\eta_{c}}^{2}=48 \frac{m_{c}^{2}}{M_{\eta_{c}}^{3}}|\Psi(0)|^{2} \\
f_{J / \Psi}^{2}=12 \frac{1}{M_{J / \Psi}}|\Psi(0)|^{2} ;
\end{gathered}
$$

therefore, the ratio $f_{\eta_{c}} / f_{J / \Psi}$ can be predicted in terms of the meson masses and of the charm quark mass:

$$
\frac{f_{\eta_{c}}}{f_{J / \Psi}}=2 m_{c}\left(\frac{M_{J / \Psi}}{M_{\eta_{c}}^{3}}\right)^{\frac{1}{2}}=0.97 \pm 0.03
$$

where the value for $m_{c}$ chosen in Sec. (2) has been used. It is known that relativistic and radiative QCD corrections could modify the prediction in Eq. (30). Using our result in Eq. (20), which includes both, and the experimental value $f_{J / \Psi}=384 \pm 14 \mathrm{MeV}$, we get:

$$
\frac{f_{\eta_{c}}}{f_{J / \psi}}=0.81 \pm 0.05
$$

A comparison of this result with that in Eq. (30) suggests that corrections are at the level of $15-20 \%$. On the other hand, Eq. (30), applied to the radial excitations $\eta_{c}^{\prime}$ and $\Psi^{\prime}$, gives the prediction: $\frac{f_{\eta_{c}^{\prime}}}{f_{\Psi^{\prime}}}=0.80 \pm 0.02$, to be compared to $\frac{f_{\eta_{c}^{\prime}}}{f_{\Psi^{\prime}}}=0.87 \pm 0.08$, obtained from Eq. (20) and using the experimental measurement $f_{\Psi^{\prime}}=282 \pm 14 \mathrm{MeV}$, possibly suggesting a minor role of radiative and relativistic corrections for these states.

Turning to nonleptonic $B$ decays, we first analyze ratios of decay widths, such as $B \rightarrow$ $K^{(*)} \eta_{c}$ and $B \rightarrow K^{(*)} \eta_{c}^{\prime}$, in the factorization approximation, where the dependence on 
the Wilson coefficient $a_{2}$ and on other weak parameters drops out, the relevant remaining dynamical quantities being the leptonic constants and the semileptonic form factors.

Consider, for example, the ratio:

$$
\tilde{R}_{K}=\frac{\Gamma\left(B^{-} \rightarrow K^{-} \eta_{c}^{\prime}\right)}{\Gamma\left(B^{-} \rightarrow K^{-} \eta_{c}\right)}=0.771\left(\frac{f_{\eta_{c}^{\prime}}}{f_{\eta_{c}}}\right)^{2}\left(\frac{F_{0}\left(M_{\eta_{c}^{\prime}}^{2}\right)}{F_{0}\left(M_{\eta_{c}}^{2}\right)}\right)^{2}
$$

where the numerical term is a phase space factor. The interesting point is that, because of the flat shape of $F_{0}\left(q^{2}\right), \tilde{R}_{K}$ mainly depends on the ratio of the leptonic constants:

$$
\tilde{R}_{K}=0.771\left(\frac{f_{\eta_{c}^{\prime}}}{f_{\eta_{c}}}\right)^{2}(1.09 \pm 0.09)^{2}=0.60 \pm 0.15 .
$$

Thus, in the factorization approximation, a measurement of $\tilde{R}_{K}$ would provide us with interesting information on $\frac{f_{\eta_{c}^{\prime}}}{f_{\eta_{c}}}$, and complement our knowledge of the properties of the $q \bar{q}$ wavefunction.

On the other hand, the analogous ratio for the decays into $K^{*}$ is given by

$$
\tilde{R}_{K^{*}}=\frac{\Gamma\left(B^{-} \rightarrow K^{*-} \eta_{c}^{\prime}\right)}{\Gamma\left(B^{-} \rightarrow K^{*-} \eta_{c}\right)}=0.381\left(\frac{f_{\eta_{c}^{\prime}}}{f_{\eta_{c}}}\right)^{2}\left(\frac{A_{0}\left(M_{\eta_{c}^{\prime}}^{2}\right)}{A_{0}\left(M_{\eta_{c}}^{2}\right)}\right)^{2}=0.381\left(\frac{f_{\eta_{c}^{\prime}}}{f_{\eta_{c}}}\right)^{2}(1.4 \pm 0.2)^{2} .
$$

Here, the ratio of the form factors deviates from unity due to the $q^{2}$-dependence of $A_{0}$. The prediction from (34) would be: $\tilde{R}_{K^{*}}=0.45 \pm 0.16$. Moreover, we observe that in the assumed factorization approximation the quantity $\sqrt{\tilde{R}_{K^{*}} / \tilde{R}_{K}}$ is sensitive to the $q^{2}$-dependence of the ratio $A_{0} / F_{0}$ :

$$
1.42 \sqrt{\frac{\tilde{R}_{K^{*}}}{\tilde{R}_{K}}}=\left(\frac{A_{0}\left(M_{\eta_{c}^{\prime}}^{2}\right) / F_{0}\left(M_{\eta_{c}^{\prime}}^{2}\right)}{A_{0}\left(M_{\eta_{c}}^{2}\right) / F_{0}\left(M_{\eta_{c}}^{2}\right)}\right)
$$

i.e. mainly to the $q^{2}$-dependence of $A_{0}$ since $F_{0}$ is predicted to have a rather flat behaviour.

A bound on the ratio

$$
R_{\eta_{c}}=\frac{\Gamma\left(B^{-} \rightarrow K^{*-} \eta_{c}\right)}{\Gamma\left(B^{-} \rightarrow K^{-} \eta_{c}\right)}=0.373\left(\frac{A_{0}\left(M_{\eta_{c}}^{2}\right)}{F_{0}\left(M_{\eta_{c}}^{2}\right)}\right)^{2}
$$

has been derived in Ref. $\left[\mathbb{8}\right.$ : $0.19 \leq R_{\eta_{c}} \leq 0.98$. This bound is satisfied by our result $R_{\eta_{c}}=$ $0.73 \pm 0.13$. In addition, for the analogous quantity $R_{\eta_{c}^{\prime}}$, we predict: $R_{\eta_{c}^{\prime}}=0.56 \pm 0.12$. 
Finally, we consider the ratio:

$$
R_{K}=\frac{\Gamma\left(B^{-} \rightarrow K^{-} \eta_{c}\right)}{\Gamma\left(B^{-} \rightarrow K^{-} J / \Psi\right)}=2.519\left(\frac{f_{\eta_{c}}}{f_{J / \Psi}}\right)^{2}\left(\frac{F_{0}\left(M_{\eta_{c}}^{2}\right)}{F_{1}\left(M_{J / \Psi}^{2}\right)}\right)^{2} .
$$

In order to predict $R_{K}$ we can use the relation: $F_{1}(0)=F_{0}(0)$, and the observation, common to the QCD sum rules calculations of the $q^{2}$-dependence of $F_{1}$, of the validity of the single pole model dominated by the $1^{-} B_{s}^{*}$ resonance [33, 35, 36]. We obtain: $R_{K}=0.94 \pm 0.25$, and, for the analogous quantity $R_{K}^{\prime}=\frac{\Gamma\left(B^{-} \rightarrow K^{-} \eta_{c}^{\prime}\right)}{\Gamma\left(B^{-} \rightarrow K^{-} \Psi^{\prime}\right)}: R_{K}^{\prime}=1.61 \pm 0.53$. This implies that, using the CLEOII experimental measurements: $\mathcal{B}\left(B^{-} \rightarrow K^{-} J / \Psi\right)=$ $(0.11 \pm 0.01 \pm 0.01) \times 10^{-2}$ and $\mathcal{B}\left(B^{-} \rightarrow K^{-} \Psi^{\prime}\right)=(0.06 \pm 0.02 \pm 0.01) \times 10^{-2}$ we expect: $\mathcal{B}\left(B^{-} \rightarrow K^{-} \eta_{c}\right)=(0.11 \pm 0.03) \times 10^{-2}$ and $\mathcal{B}\left(B^{-} \rightarrow K^{-} \eta_{c}^{\prime}\right)=(0.10 \pm 0.05) \times 10^{-2}$, and therefore these decays are in a range well-accessible to the present experimental facilities.

With these predictions we conclude our analysis. The relatively large decay rates of the processes $B \rightarrow K^{(*)} \eta_{c}$ and $B \rightarrow K^{(*)} \eta_{c}^{\prime}$ should allow their observation in the near future. This measurement will shed more light on the problem of factorization, which is a basic assumption in the present analysis of heavy meson nonleptonic decays.

From the theoretical point of view, it should be interesting to compare the results obtained here with other QCD calculations. For example, it should be possible to calculate the leptonic constants of $\eta_{c}$ and $\eta_{c}^{\prime}$ mesons by lattice QCD. Moreover, regarding the $B \rightarrow K^{(*)}$ form factors, a possible independent test of the $q^{2}$-dependence could be obtained, e.g., by using light-cone sum rules. Also, extrapolations of form factors in $q^{2}$ and in the heavy quark mass, starting from the charm mass, in the lattice QCD framework [37], could possibly provide us with enough information, at least at a qualitative level, to be compared with the QCD sum rules results. 


\section{Acknowledgments}

We would like to thank F.Buccella, F.De Fazio, G.Nardulli and P.Santorelli for interesting discussions. The work of (NP) was supported in part by the HCM, EEC Contract ERBCHRXCT930132. The work of (CAD) was supported in part by the Foundation

for Research Development (ZA) and the John Simon Guggenheim Memorial Foundation (USA). 


\section{References}

[1] M. Gourdin, A.N. Kamal and X.Y. Pham, Phys. Rev. Lett. 73 (1994) 3355.

[2] R. Aleksan, A. Le Yaouanc, L. Oliver, O. Pene and J.C. Raynal, Report DAPNIA/SPP/94-24, LPTHE-Orsay 94/15.

[3] For a review see: T.E. Browder, K. Honsheid and S. Playfer, in: "B decays", S. Stone Ed., World Scientific, 1994.

[4] G. Altarelli, G. Curci, G. Martinelli and S. Petrarca, Nucl. Phys. B 187 (1981) 461;

A.J. Buras, M. Jamin, M.E. Lautenbacher and P.E. Weisz, Nucl.Phys. B 370 (1992)

69 and Nucl.Phys. B 375 (1992) 501 (addendum);

M. Ciuchini, E. Franco, G. Martinelli and L. Reina, Nucl. Phys. B 415 (1994) 403.

[5] A.J. Buras, Report MPI-PhT/94-60, TUM-T31-75/94.

[6] M. Wirbel, B. Stech and M. Bauer, Z. Phys. C 29 (1985) 637.

[7] M. Gourdin, Y.Y. Keum and X.Y. Pham, Report PAR/LPTHE/95-01.

[8] M. Gourdin, Y.Y. Keum and X.Y. Pham, Report PAR/LPTHE/94-32.

[9] A.N. Kamal and A.B. Santra, Report Alberta Thy-27-94 and Alberta Thy-31-94;

C.E. Carlson and J. Milana, Report WM-94-110;

H.-Y. Cheng and B.Tseng, Report IP-ASTP-21-94.

[10] G. 't Hooft, Nucl. Phys. B 72 (1974) 461;

E. Witten, Nucl. Phys. B 149 (1979) 285.

[11] M. Dugan and B. Grinstein, Phys. Lett. B 255 (1991) 583.

[12] U. Aglietti, Phys. Lett. B 292 (1992) 424.

[13] B. Block and M.A. Shifman, Sov. Jour. Nucl. Phys. 45 (1987) 135, 301, 522; Nucl. Phys. B 389 (1993) 534, Nucl. Phys. B399 (1993) 441, 459. 
[14] A. Khodjamirian and R. Rückl, Report MPI-PhT/94-26, LMU 05/94.

[15] M. Bauer, B. Stech and M. Wirbel, Z. Phys. C 34 (1987) 103.

[16] M.A. Shifman, A.I. Vainshtein and V.I. Zakharov, Nucl. Phys. B 147 (1979) 385,448; L.J. Reinders, H. Rubinstein and S. Yazaki, Phys. Rep. 127 (1985) 1.

[17] N.G. Deshpande and J. Trampetic, Phys. Lett. B 339 (1994) 270.

[18] V.A. Beilin and A.V. Radyushkin, Nucl. Phys. B 260 (1985) 61.

[19] D. J. Broadhurst and S. C. Generalis, Open University Report No. OUT-4201-8 (1982);

S. C. Generalis, PhD thesis OUT-4102-13 (Open University, 1983);

see also: K. Schilcher, M.D. Tran and N. F. Nasrallah, Nucl. Phys. B 181 (1981) 91; B 187 (1981) 594 (E).

[20] R.A. Bertlmann et al. Z. Phys. C 39 (1988) 231;

C.A. Dominguez and J. Solà, Z. Phys. C 40 (1988) 63.

[21] C.A. Dominguez, G.R. Gluckman and N. Paver, Phys. Lett. B 333 (1994) 184;

S. Narison, Report CERN/TH-7444/94, to appear in the Proceedings of the "QCD94" Workshop, Montpellier (France) 7-13 July 1994.

[22] C. Edwards et al., Phys. Rev. Lett. 48 (1982) 70.

[23] R.A. Bertlmann, Nucl. Phys. B 204 (1982) 387.

[24] M. Abramowitz and I.A. Stegun, Handbook of Mathematical Functions (Dover, New York, 1972).

[25] V.A. Novikov et al., Phys. Rev. Lett. 38 (1977) 626; Phys. Lett. B 67 (1977) 409; Phys. Rep. 41 (1978) 1.

[26] L.J. Reinders, H. Rubinstein and S. Yazaki, Phys. Lett. B 113 (1982) 411. 
[27] M. Shifman, JETP Lett. 30 (1979) 546.

[28] B.L. Ioffe and A.V. Smilga, Nucl. Phys. B 216 (1983) 373;

A.V. Nesterenko and A.V. Radyushkin, Phys. Lett. B 115 (1982) 410.

[29] For a review see the reprints volume: "Vacuum structure and QCD Sum Rules", edited by M.A. Shifman, North-Holland, 1992.

[30] C.A.Dominguez and N.Paver, Phys. Lett. B 293 (1992) 197.

[31] C.A.Dominguez, C. van Gend and N.Paver, Phys. Lett. B 253 (1991) 241;

K.G.Chetyrkin, C.A.Dominguez, D.Pirjol and K.Schilcher, Report MZ-TH/94-21 (1994);

M.Jamin and M.Münz, Report CERN-TH.9435/94 (1994).

[32] C.A.Dominguez, in Proceedings of the Third Workshop on the Tau-Charm Factory, Marbella, Spain, 1993, edited by J.Kirkby and R.Kirkby, Editions Frontieres, Gifsur-Yvette, France, 1994.

[33] P. Ball, V. Braun and H.G. Dosch, Phys. Rev. D 44 (1991) 3567.

[34] P. Colangelo, F. De Fazio and P. Santorelli, Report BARI-TH/94-174, to appear in Phys.Rev.D.

[35] P. Colangelo and P. Santorelli, Phys. Lett. B 327 (1994) 123.

[36] P. Ball, Phys. Rev. D 48 (1993) 3190;

V.M. Belyaev, A. Kodjamirian and R. Rückl, Z.Phys C 60 (1993) 349.

[37] A.Abada et al., Nucl. Phys. B416 (1994) 675;

K.C.Bowler et al., UKQCD Collaboration, Edinburgh Preprint 94/546, Southampton Preprint SHEP 93/94-32. 


\section{FIGURE CAPTIONS}

Fig. 1

The mass of the $\eta_{c}$ from Hilbert moments as a function of $n$ for $\Lambda=200 \mathrm{MeV}, m_{c}=$ $1.39 \mathrm{GeV}$, and $<\alpha_{s} G^{2}>=0.063 \mathrm{GeV}^{4}$. The solid curve is the prediction and the dash curve the experimental value.

\section{Fig. 2}

The leptonic decay constant $\tilde{f}_{\eta_{c}}=f_{\eta_{c}} / \sqrt{2}$, using a single pole spectral function in Hilbert moments, as a function of $n$ and for the same values of input parameters as in Fig. 1. The solid (dash) curve is obtained using the predicted (experimental) value of $M_{\eta_{c}}$.

\section{Fig. 3}

The mass of the $\eta_{c}$ from the Laplace transform for $\Lambda=200 \mathrm{MeV}, m_{c}=1.39 \mathrm{GeV}$, and $\left\langle\alpha_{s} G^{2}>=0.063 \mathrm{GeV}^{4}\right.$. The solid curve is the prediction and the dash curve the experimental value.

\section{Fig. 4}

The leptonic decay constant $\tilde{f}_{\eta_{c}}=f_{\eta_{c}} / \sqrt{2}$, using a single pole spectral function in the Laplace transform, and for the same values of input parameters as in Fig. 3. The solid (dash) curve is obtained using the predicted (experimental) value of $M_{\eta_{c}}$. 


\section{Fig. 5}

The form factor $F_{0}\left(q^{2}\right)$ for the transition $B \rightarrow K$. The curves refer to the sets of parameters: $s_{0}=33 \mathrm{GeV}^{2}$ and $s_{0}^{\prime}=1.3 \mathrm{GeV}^{2}$ (continuous line), $s_{0}=33 \mathrm{GeV}^{2}$ and $s_{0}^{\prime}=1.5 \mathrm{GeV}^{2}$ (dashed line), $s_{0}=36 \mathrm{GeV}^{2}$ and $s_{0}^{\prime}=1.3 \mathrm{GeV}^{2}$ (dotted line), $s_{0}=36 \mathrm{GeV}^{2}$ and $s_{0}^{\prime}=1.5 \mathrm{GeV}^{2}$ (dashed-dotted line). The Borel parameters in the $B$ and $K$ channel are fixed to $M^{2}=8 \mathrm{GeV}^{2}, M^{2}=2 \mathrm{GeV}^{2}$, respectively.

\section{Fig. 6}

The form factor $A_{0}\left(q^{2}\right)$ for the transition $B \rightarrow K^{*}$. The curves refer to the sets of parameters: $s_{0}=33 \mathrm{GeV}^{2}$ and $s_{0}^{\prime}=1.5 \mathrm{GeV}^{2}$ (continuous line), $s_{0}=33 \mathrm{GeV}^{2}$ and $s_{0}^{\prime}=$ $1.7 \mathrm{GeV}^{2}$ (dashed line), $s_{0}=36 \mathrm{GeV}^{2}$ and $s_{0}^{\prime}=1.5 \mathrm{GeV}^{2}$ (dotted line), $s_{0}=36 \mathrm{GeV}^{2}$ and $s_{0}^{\prime}=1.7 \mathrm{GeV}^{2}$ (dashed-dotted line). The Borel parameters in the $B$ and $K^{*}$ channel are the same as in Fig.5. 
This figure "fig1-1.png" is available in "png" format from: http://arxiv.org/ps/hep-ph/9502374v1 
This figure "fig1-2.png" is available in "png" format from: http://arxiv.org/ps/hep-ph/9502374v1 
This figure "fig1-3.png" is available in "png" format from: http://arxiv.org/ps/hep-ph/9502374v1 